\title{
Approaches to treatment of interstitial lung disease
}

\author{
MARGARET E. H. TURNER-WARWICK \\ Ph.D., M.A., D.M., F.R.C.P.
}

Brompton Hospital, London SW3

\section{Introduction}

This short article will not attempt to detail every treatment possibility in each of the 150 or so interstitial lung diseases. Rather it will highlight some of the principles which, if followed, improve management. It will also highlight some of the continuing problems in this very difficult area of respiratory medicine.

\section{The importance of establishing a correct diagnosis}

It might appear self evident that treatment can only be suitable when the correct diagnosis has been established. In practice, this principle is often ignored. The probable explanation to this is that certain interstitial diseases are a great deal more common than others and it is tempting to be satisfied with a clinical likelihood rather than objective proof. However as the student said to the examiner when chastised for making a diagnosis because it was too rare, he replied, 'rare diseases would occur more often if they were diagnosed more frequently'! In particular because of its common occurrence in young people, sarcoidosis is often diagnosed and treated on insufficient evidence and only later, when the patient has deteriorated seriously is the correct diagnosis apparent. This has important therapeutic implications if, for example, the patient in fact suffered from alveolar proteinosis where a large volume lung lavage can be life-saving.

The second reason why a correct diagnosis is important is that the natural history of different diseases varies strikingly and knowledge of this is essential for logical planned treatment. For example some $70 \%$ of patients with sarcoidosis resolve spontaneously within about 2 years while $50 \%$ of patients with cryptogenic fibrosing alveolitis are dead within 4 or 5 years.

\section{The importance of staging}

Clearly patients with chronic interstitial disease present at very different stages of the disease and opportunities for treatment are obviously greatest at an earlier stage when there is more inflammation and less established fibrosis. Thus staging of disease becomes as important as diagnosis. For both of these reasons I personally have a strong preference for obtaining lung tissue before treatment wherever possible. Obviously this rule should be modified in very elderly patients or the very sick. However too many patients have been allowed to get very sick before the physician in charge appreciates the seriousness of the condition. A particular feature of advanced disease of any cause (including druginduced interstitial disease or that due to organic dusts) is that there appears to be a point of no return even after removal from the initiating agent. At this stage not only is there no response to corticosteroids but the disease may progress relentlessly without further exposure.

\section{What type of biopsy}

Where a pathognomonic pathology is expected from the clinical features, then a transbronchial biopsy may be adequate (this is often all that is necessary in patients with sarcoidosis, idiopathic haemosiderosis, alveolar proteinosis, acute extrinsic allergic alveolitis or pneumocystis pneumonia). In sarcoidosis a bronchial mucosal biopsy is often positive and establishes the diagnosis satisfactorily.

However when the clinical features suggest that the pathology may be less clear cut my own current preference is to request an open biopsy performed through a small sub-mammary incision. There is considerable advantage in asking a co-operative surgeon to inspect the lung and obtain two samples one from the least and one from the most affected portion, to ensure haemostasis and the absence of air leaks before closure. Good results have also been obtained with the Steel trephine drill but many physicians find the instrument difficult to use. Various forms of needle biopsy have been and continue to be advocated but the samples are relatively small, the site cannot be selected by palpation and uncontrolled disasters have occurred in most series sooner or later. 
Clearly open surgery is also potentially dangerous if case selection or postoperative care is inadequate. Patients on corticosteroids seem to have a greater chance of complications than those not on steroids which is an additional reason for performing a biopsy before treatment is started.

The argument is always put forward that since corticosteroids are the only treatment available an empirical trial is a practical and simple way to proceed. This policy in practice leads physicians into great difficulties. When the patient has a doubtful response, the physician is then in a quandary with regard to the diagnosis and so no prognostic indications can be given to the patient or their relatives. In certain instances other specific treatments are available (e.g. large volume lavage in alveolar proteinosis and perhaps hormone treatment in lymphangioleiomyomotosis, plasma pheresis in some types of pulmonary haemorrhage and high dose cotrimoxazole in pneumocystis pneumonia).

Further, where there is not too much irreversible fibrosis, immunosuppressive drugs such as cyclophosphamide or azathioprine have been shown to be a real alternative when corticosteroids fail; but such drugs have serious potential side effects and should only be used when the condition is appropriate.

\section{A plan of monitoring response}

Responses to treatment are often at best partial so that a systematic plan should be made not only with regard to drug dosage but also as to how the response is to be monitored. The most practical current tools include systematic semi-quantitative grading of the patients' symptoms, the chest radiograph and standard lung function tests. The latter should include both lung volume and transfer factor measurements for carbon monoxide because these two measurements do not always change in parallel. Our own practice is also to undertake serial standardized exercise tests with blood gas measurements, but whether this gives much additional information is still to be examined formally. We also include a 6minute walking test as well as the patient's own assessment of breathlessness on a questionnaire and a $10 \mathrm{~cm}$ analogue scale. These records are simple to keep and are probably more accurate than simple narrative statements. However formal validation has not yet been undertaken.

\section{Plan of dosage schedule}

When corticosteroids are used (and irrespective of the disease) it is probably reasonable to start with a fairly large dose. Our normal practice is to use about $40 \mathrm{mg}$ of prednisolone in sarcoidosis (where the disorder tends to respond fairly easily) and $60 \mathrm{mg}$ in cryptogenic fibrosing alveolitis where the condition is usually less responsive. This relatively high dose is气 used for about 4 weeks to achieve optimal improve $\frac{\pi}{8}$ ment in as many parameters as possible. Havingo achieved maximum response for an individual patient (which is often far from complete resolution of disease) the dose can be titrated to the lowest which maintains optimal improvement. We label (green by our code) the X-ray showing maximum improvemen so that this film can be compared with subsequen ones and we believe it is the easiest way of detecting insidious deterioration which may occur so slowlyes that it cannot be detected by comparing any two sequential films.

In sarcoidosis a number of patients can be main $\vec{\omega}$ tained on an alternate day regime. However about कs third of our patients deteriorate on such a regime an $\&$ a daily dose is then used. We give the whole doseonce a day in the morning.

\section{Special considerations in individual disorders}

\section{Sarcoidosis}

Indications for treatment of interstitial pulmonary lesions in sarcoidosis present special problems in relation to the natural history of the condition. Most authors agree that overall some $70 \%$ of all patient with interstitial radiographic infiltrates will resolve spontaneously and without treatment within abou甲 200 years. Others remit more slowly over $2-8$ years tift the longer resolution takes the greater the chancespop some irreversible scarring developing. This in itselk may be unimportant in some cases although secon dary infection from anatomical distortion or aspergil lomas in disorganized lung may be responsible fo $\bar{D}$ both morbidity and mortality. The physician is faced with a dilemma because on the one hand the treatment is often unnecessary, but if delayed too long, the disease may compromise both health an even life. Cases presenting with interstitial infiltrates without erythema nodosum or hilar node enlarge 3 ment especially in older individuals (over the age of about 30 years) are probably at special risk of developing progressive disease.

Formal clinical trials have so far failed to demon strate definite benefits of steroids mainly becauses they have not been designed bearing in mind the natural history of the sub-populations studied. Treate ment for the time being thus has to be based on a logical, if unproven basis. Our present policy is outlined, recognizing that many do not agree and that we, like others, have no proof that the policy wen have adopted is correct.

Unless there are over-riding indications for treat ment (e.g. rapid deterioration of breathlessness of

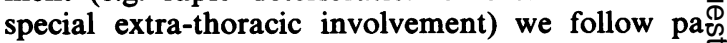
tients for about 1-2 years from their first presentation but do not treat with steroids, to allow them . 
maximum opportunity of resolving spontaneously. If however the radiograph fails to improve over this period, our principle is to start corticosteroid treatment even in the absence of symptoms or abnormal lung function tests. The policy is thus to avoid treating patients who will resolve spontaneously but to intervene before irreversible changes have developed. In practice, this balance point is hard to achieve but the principle is clear.

Once treatment is started we aim to obtain maximum improvement in symptoms, chest radiographic appearances, lung volumes and carbon monoxide diffusion capacity (DLCO) and to maintain this on the lowest possible dose of steroids. In practice this is achieved on a maintenance alternate day regime in about two-thirds of our patients using between 10 and $15 \mathrm{mg}$ of prednisolone. In the remaining third a daily regimen on about the same dose is necessary.

We maintain patients on a minimal dose for about 1 year then and attempt to stop-always explaining to the patient that treatment may have to be restarted if the X-ray deteriorates.

Relapse may not occur immediately on withdrawal of corticosteroids so patients have to be watched particularly carefully over the years following discontinuation of therapy. We have seen late relapses occurring after 3 or 4 years.

Relapse of sarcoidosis while under treatment is so often due to two correctable factors. The patient may not be taking the prescribed dose or indeed any steroids at all, or the doctor may be continuing to prescribe an arbitrary maintenance dose when the disease is obviously relapsing. Individual titration of the dose against maximum response is a logical policy.

Clearly validation of a particular treatment policy in sarcoidosis is very difficult in view of the widely varying course of disease, its often slow rate of change and the inadequacy of accurate markers of activity. However it should be remembered that once fibrosis has developed the consequences are usually compromising and may be directly or indirectly lethal.

\section{Cryptogenic fibrosing alveolitis}

Management of cryptogenic fibrosing alveolitis in some ways presents fewer problems but in others is more difficult. The decision when to treat is easier because the outlook is so much worse $(50 \%$ of patients dead 4-5 years); thus early treatment to obtain maximum benefit is less controversial. However response to steroids is less frequent and in responders is usually only partial, so that titration of the drug dosage is more difficult.

We have shown that the factors tending to predict a better response to steroids include younger patients, women, less radiographic abnormality, shorter duration of disease combined with a more severe reduction of forced vital capacity (less than $66 \%$ predicted). Those with more cellular biopsies and less established fibrosis, those with abnormal numbers of lymphocytes in the lavage sample and those with type III collagen on the biopsy all do better. However all of these predictors are identified by comparison of groups of patients and there are many individuals who are exceptions to the rules. In particular we have seen a number of patients with an initial increase in the neutrophils in the lavage who in fact respond to corticosteroids. These individuals are interesting because serial lavage samples often show a fairly rapid return towards normal.

The principle of management with steroids follows the general plan outlined above of using a fairly high dose initially to obtain maximum response. We use around $60 \mathrm{mg}$ of prednisolone for one month titrating to a lower maintenance dose around $20 \mathrm{mg}$ daily or on alternate days if maximum radiographic, clinical and physiological improvement is achieved.

Where corticosteroids fail, cyclophosphamide in a dose of about $2 \mathrm{mg} / \mathrm{kg} /$ day may be successful. We have now had several carefully monitored patients where this is the case. Improvement tends to occur slowly over 3-6 months and we normally continue for this time before accepting treatment failure. It is our principle to do regular blood counts every week or two throughout the course of immunosuppressant therapies. If there is any evidence of haematuria it is our current practice to change to azathioprine. On the dosage used we have not seen serious haemorrhagic cystitis or alopecia. Attempts to arrest disease which have failed to respond either to steroids or cyclophosphamide have been made using penicillamine. While, in occasional patients, the disease seems to have been arrested, this experience remains anecdotal at the present time and substantial trials have not yet proved its value.

Some patients have a component of airflow obstruction and a few of these get improvement with nebulized salbutamol. As the disease advances patients are apt to develop recurrent infections and these should be treated with antibiotics.

The challenge in cryptogenic fibrosing alveolitis is to identify cases at an earlier stage. However the disease is often so insidious, occurring in relatively sedentary individuals of later middle age that symptoms are only reported when the disease is already relatively advanced. Full assessment at an early stage is however in our view imperative if better results are to be obtained.

\section{Conclusion}

Therapy of interstitial lung disease presents a major challenge because the disorders have very 
variable natural courses, measurement of disease activity is often difficult and improvement on drug therapy is often limited. Many of the conditions to be treated are relatively uncommon so that adequate clinical trials are difficult to design and the patient populations are very heterogeneous.
In spite of these difficulties patients with these disorders have a serious long-standing problem which if mishandled will lead to compromised health and in some instances will shorten their lives. Thus: the most careful assessment is mandatory if an progress is to be made. 